\title{
CAPABILITY OF MATTERPORT 3D CAMERA FOR INDUSTRIAL ARCHAEOLOGY SITES INVENTORY
}

\author{
R. Shults ${ }^{1 *}$, E. Levin ${ }^{1}$, R. Habibi ${ }^{1}$, S. Shenoy ${ }^{1}$, O. Honcheruk ${ }^{2}$, T. Hart ${ }^{1}$, Z. An ${ }^{1}$ \\ ${ }^{1}$ School of Technology, Michigan Technological University, Townsend Drive, Houghton, USA - rshults@mtu.edu, \\ elevin@mtu.edu,rhabibi@mtu.edu, sanjaysh@mtu.edu, thart@mtu.edu, zan1@mtu.edu \\ ${ }^{1}$ GIS and Land Management Faculty, Kyiv National University of Construction and Architecture, Povitroflotskij Ave, Kyiv, Ukraine \\ -h.olesya13@ukr.net
}

Commission V, WG V/7

KEY WORDS: Industrial Archaeology, Calibration, Scale Factor, the Quincy Mine, Indoor Modelling, Hoist Engine

\begin{abstract}
:
This paper provides an overview of state-of-the-art technology and sensor for the inventory of industrial archaeology. As an object of study, a historical copper shaft the Quincy Mine in Michigan State Upper Peninsula was chosen. This shaft was operated for nearly 100 years from 1846 to 1945 and today, what remains of the shaft is a part of the Keweenaw National Historical Park. The main sensor for data acquisition that was used is a 3D camera Matterport. In given research, the results of the above ground scanning using of Matterport are presented. Before scanning a calibration of Matterport camera was performed. The calibration was done by comparison of coordinates of targets determined by FARO Scanner. It was found out that the camera has significant systematic errors which have to be accounted during data processing. Because of the specific data structure of Matterport camera, only the scale factor was calculated and then applied to point clouds. Field works comprised historical and archive data collection and analysis, reconnaissance and scanning of the Quincy Mine interior. As a final result of the research 3D model of the Quincy Mine interior was constructed. Further, this model is going to be used for the Quincy Mine Museum virtual tours. There are many advantages to using Matterport. First of all, such a technology does not need dense geodetic support, average working time at station equals to 2-3 minutes. Cost of Matteroprt equipment is competitive to the cheapest terrestrial laser scanners.
\end{abstract}

\section{INTRODUCTION}

A long time has gone since fundamental work dedicated to industrial archaeology its methods (Palmer, 1989) was published. There the most conventional geodetic methods for industrial archaeology objects documentation were described. Since that, many changes in the field of geospatial technologies have been happened. There is no need to discuss and describe all those inventions and developments, but we want to point out on the main technologies and devices, which have led to the new paradigm of industrial archaeology sites inventory and documentation. Among them: brand-new, low-cost digital cameras, terrestrial and aerial laser scanning, ease-of-use and high-precision GNSS, unmanned aerial vehicles, cameraequipped total stations, etc. All of those technologies or their combinations allow performing inventory both on a global and local scale. Many authors have been applying those technologies since their development for industrial archaeology tasks, a couple of good examples can be found in (Agostinelli, et al. 2007., Gruenkemeier, 2008., Costantino, et al. 2010., Barazzetti, et al. 2018).

From time to time, new start-up projects for 3D data acquisition begin which are brought about by new technological developments. Matterport Pro 3D Camera, further in the paper Matterport (Matterport, 2019a) is a good example one of such a project. As the main purpose of Matterport is data acquisition for indoor modelling, some researches have been done (Virtanen, et al. 2018, Gärdin, et al. 2018). In the first paper, the opportunity of Matterport for the creation of interactive virtual environments has been studied. The second one is not only a good example of
Matterport application but also presents an attempt to assess data accuracy. Since the camera was developed, it forced paper authors' to use it for industrial archaeology tasks. The idea of using Matterport for industrial archaeology is quite new. In order to check Matterport capability for this task, the Quincy Mine historical object was chosen. As a subject of indoor modeling, the Quincy Mine Hoist Engine was chosen. The object satisfies extreme requirements, insofar as the scanning conditions are far from optimal from the point of view of distances, light conditions, and the temperature range. A new sensor always needs accuracy study, so that, before data capturing and modelling, the sensor check and calibration have to be done.

One of the goals of the research was a support of the Quincy Mine Hoist Association (QMHA), a local volunteer organization whose goal is to preserve and restore the history of the Quincy Mine. Of interest an integration Matterport data with other 3D data with the aim of creating a complex $3 \mathrm{D}$ model, which is joining topographical data, outdoor building model and indoor model. This integration will be the last step of Matterport workflow for industrial archaeology sites inventory.

\section{MATTERPORT PRO 3D CAMERA}

Here we presented a brief description of Matterport (Fig. 1) and its parameters (Matterport, 2019b). As it pointed out in (Pulcrano, et al., 2019), the basic principle of the system is SLAM technology (Simultaneous Localization and Mapping). The system uses a structured light (infrared) 3D sensor with capture time equals to 20_seconds per scan.

\footnotetext{
* Corresponding author
} 


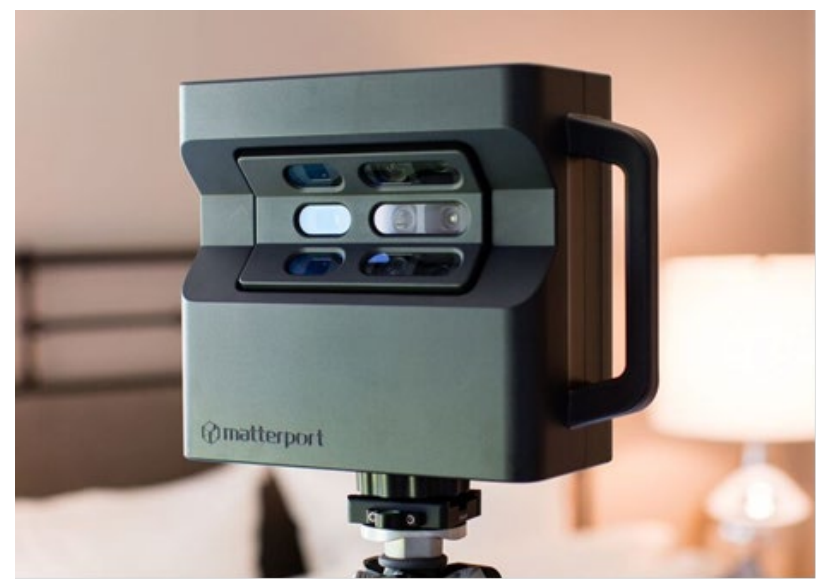

Figure 1. Matterport Pro 3D Camera (Matterport, 2019b)

Maximum operating distance is $4.5 \mathrm{~m}$. The important parameter is a resolution, which for the camera equals to 10 points per degree or 3600 points at equator, 1800 points at meridian, which in total 4 million points per pano. A typical field of view equals to $360^{\circ}$ (horizontal) $\times 300^{\circ}$ (vertical). A more detailed description a reader can find in (Lehtola, et al. 2017, Gärdin, et al. 2018, Pulcrano, et al., 2019).

\section{MATTERPORT PRO 3D ACCURACY CHECK AND CALIBRATION}

\subsection{Related works}

Presumably, for such a camera type a detailed accuracy analysis for the first time was considered in (Khoshelham et al. 2012). Since that time, many publications were dedicated to accuracy check and calibration. Having studied state-of-the-art publications was found out that the most common way to check the accuracy of Matterport is a comparison of point clouds.

The Matterport data quality question was considered in (Lehtola, et al. 2017, Gärdin, et al. 2018, Pulcrano, et al., 2019). The first one discusses a comparison of different indoor modeling systems, among them Matterport. Authors perform point cloud to point cloud comparison and as a quality measure suggested their new metric. As error-free data, TLS point clouds were chosen. The main conclusion is that Matterport data have a low accuracy level despite on good photo-realistic quality. They assessed Matterport accuracy equals to $70 \mathrm{~mm}$ towards vertical. More comprehensive research has been presented by (Gärdin, et al. 2018). One of the advantages of the research is an application of Matterport data to real objects (bridges), which can be treated as industrial archaeology objects. Authors tried to compare distances, which were got from TLS point cloud and close-range photogrammetry modelling with Matterport distances. The problem is that authors should have done these measurements to targets and not to artificial contours, i.e., corners, edges, planes, etc. Such measurements were distorted by bad contours recognition on point clouds and have led to unreliable results in many cases. Anyway, authors declare Matterport accuracy in a range of $0.3-3 \%$ versus TLS data. In (Pulcrano, et al., 2019) authors used ICP algorithm to compare TLS and close-range photogrammetry point cloud with Matterport data. It is clear that these data have different structure and density, which in turn can lead to some problems with clouds orientation. As a result of such a comparison, authors declared the following accuracy: $80 \mathrm{~mm}$ in a horizontal plane and $150 \mathrm{~mm}$ in vertical plane respectively. Unfortunately, no one has noticed on a probable systematic error in their results. That is why before calibration we have decided to check our Matterport data for any systematic errors availability. Tailored to the measurement principle of Matterport the main attention has to be paid to a scale distortion.

\subsection{Data Collection}

In order to check Matterport data for systematic errors availability, the following test field has been organized. Room with dimensions $12 \times 9 \times 3.2 \mathrm{~m}$ has been chosen. Totally, 21 welldistributed (at different heights) control targets were placed on the room walls. For better control, coordinates of these control targets were determined by precise Topcon total station. Then, terrestrial laser scanning by FARO Scanner of the room from six scan stations has been performed (Fig. 2).

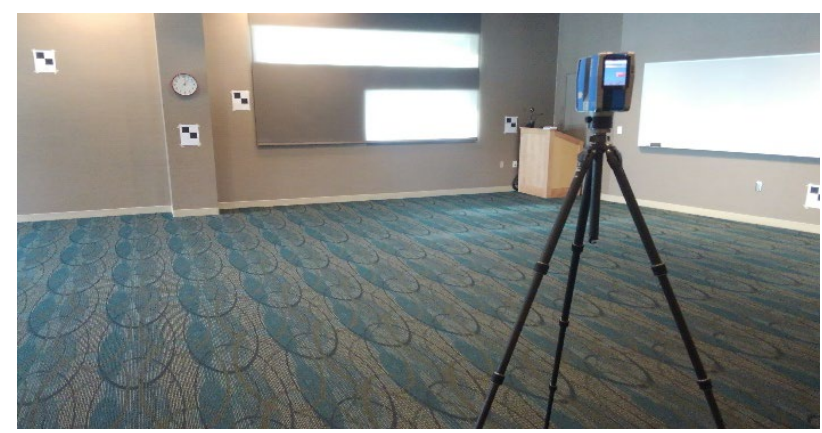

Figure 2. Test field scanning by FARO Scanner

After the scans orientation in one model, the total size of the model was equal over 40 million points (Fig. 3).

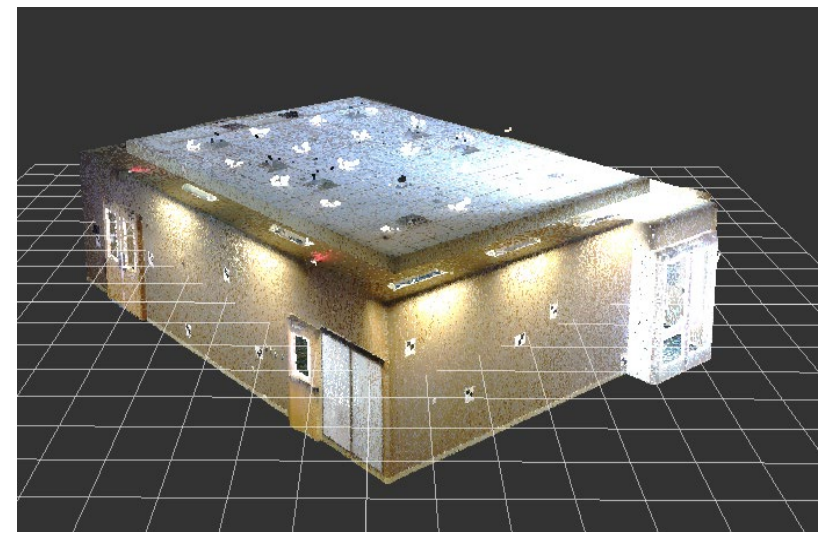

Figure 3. Point cloud of the test field with control targets This preparation step took 45 minutes.

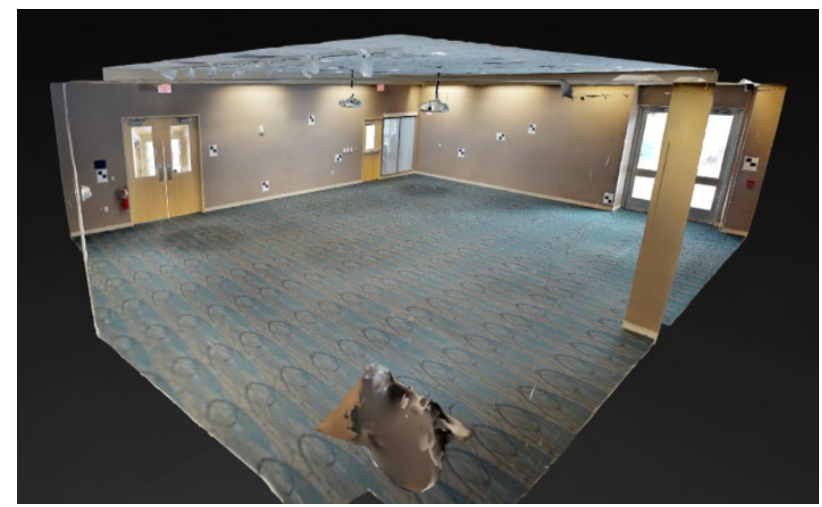

Figure 4. Matterport 3D model of the test field with control targets

At the next step, Matterport surveying of the same room has been done. Surveying by Matterport from eight stations with model size over 4 million points has been accomplished (Fig. 4). It is 
important to mention that the data capturing by Matterport took just 10 minutes. These data TLS and Matterport were used at the next step for a systematic error revealing.

\subsection{Accuracy Check - Distances Comparison}

It was mentioned that the main attention, have to be paid to a scale distortion. The easiest way is to find the scale error by distances comparing. To this aim, by Matterport and TLS coordinates of control targets, totally 210 distances were calculated, and appropriate differences were found. These differences are presented in Fig. 5.

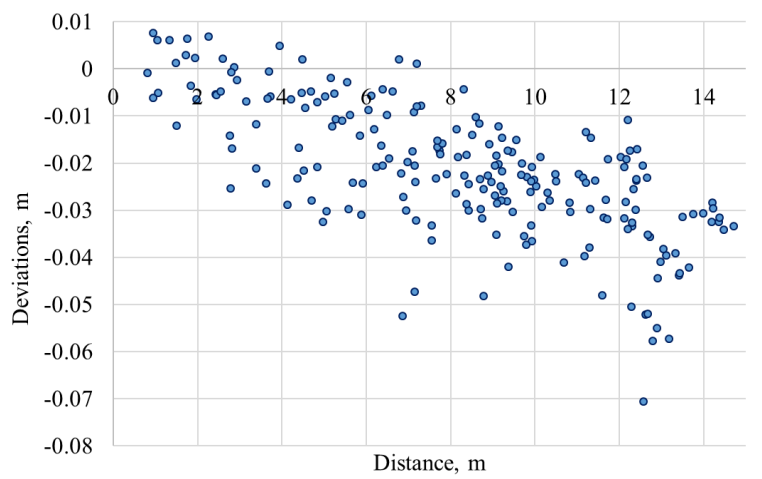

Figure 5. Distances deviations Matterport vs. FARO Scanner

It is clear that the differences distribution has a strong correlation with distances, the longer the distance, the bigger the difference, in general. The differences distribution has mean value -0.022 $\mathrm{m}$ and root mean square error $0.014 \mathrm{~m}$. These data have proved our suggestion about the scale distortion of Matterport data. By these differences, we can calculate the scale factor to account the systematic error. Finally, we got the scale factor equals to0.997.

The scale factor was applied to calculated distances and as a result, mean value became equals to $-0.001 \mathrm{~m}$ and root mean square error $-0.010 \mathrm{~m}$. The differences distribution after scale factor accounting is presented in Fig. 6.

There is no doubt that after the scale correction the distances differences have a random distribution in general. Of course, it is not necessarily Gauss distribution, but anyway without remarkable systematic error.

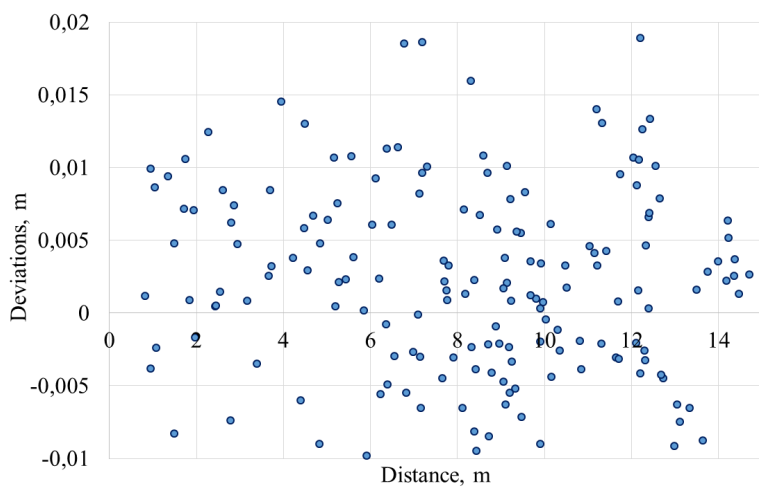

Figure 6. Distances deviations after the systematic error correction

Once the availability of systematic error is determined, the calibration has to be performed.

\subsection{Calibration}

Under calibration (Khoshelham et al. 2012) understands the determination of a conventional set of camera parameters and particular parameters, namely the base length and the reference distance. Due to a specific data structure of Matterport, i.e. point cloud, we suggest using a classical approach from TLS calibration, which is based on 7-parameter Helmert transformation. The most significant effect has a scale factor. Insofar as we consider only the scale factor, it is possible to confine by the simple model without additional parameters. In order to find transformation parameters Java'Applied·Geodesy·3D (JAG3D, 2019) software has been used. Results of calculations are presented in Table 1.

\begin{tabular}{|c|c|c|c|}
\hline Parameter & Value & $\sigma$ Par & Test \\
\hline Tx & $-5.8489 \mathrm{~m}$ & $1.7 \mathrm{~mm}$ & $\mathbf{X}$ \\
\hline Ty & $4.5493 \mathrm{~m}$ & $1.7 \mathrm{~mm}$ & $\mathbf{X}$ \\
\hline $\mathrm{Tz}$ & $1.6798 \mathrm{~m}$ & $1.9 \mathrm{~mm}$ & $\mathbf{X}$ \\
\hline $\mathrm{q} 0$ & 0.00627075 & 0.00019476 & $\mathbf{X}$ \\
\hline $\mathrm{q} 1$ & -0.90408884 & 0.00005095 & $\mathbf{X}$ \\
\hline $\mathrm{q} 2$ & -0.42729374 & 0.00010780 & $\mathbf{X}$ \\
\hline $\mathrm{q} 3$ & -0.00202597 & 0.00015138 & $\mathbf{X}$ \\
\hline $\mathrm{a} 11$ & 0.99736325 & 0.00023678 & $\mathbf{X}$ \\
\hline $\mathrm{a} 12$ & 0.00000000 & 0.00000000 & $\checkmark$ \\
\hline $\mathrm{a} 13$ & -0.00000000 & 0.00000000 & $\mathbf{X}$ \\
\hline $\mathrm{a} 22$ & 0.99736325 & 0.00023678 & $\mathbf{X}$ \\
\hline $\mathrm{a} 23$ & 0.00000000 & 0.00000000 & $\checkmark$ \\
\hline $\mathrm{a} 33$ & 0.99736325 & 0.00023678 & $\mathbf{X}$ \\
\hline $\mathrm{M}$ & $-2636.7 \mathrm{~mm} / \mathrm{km}$ & $236.8 \mathrm{~mm} / \mathrm{km}$ & $\mathbf{X}$ \\
\hline $\mathrm{Rx}$ & 199.16791 gon & $25.01 \mathrm{mgon}$ & $\mathbf{X}$ \\
\hline $\mathrm{Ry}$ & 0.10794 gon & $19.00 \mathrm{mgon}$ & $\mathbf{X}$ \\
\hline $\mathrm{Rz}$ & 56.21379 gon & $15.18 \mathrm{mgon}$ & $\mathbf{X}$ \\
\hline
\end{tabular}

Table 1. Estimated transformation parameters and its uncertainties

\begin{tabular}{|c|c|c|c|c|c|c|}
\hline Point & $\varepsilon X, \mathrm{~mm}$ & $\varepsilon \mathrm{Y}, \mathrm{mm}$ & $\varepsilon \mathrm{zZ}, \mathrm{mm}$ & $\begin{array}{c}\sigma \mathrm{X}, \\
\mathrm{mm}\end{array}$ & $\begin{array}{c}\sigma \mathrm{Y}, \\
\mathrm{mm}\end{array}$ & $\begin{array}{c}\sigma \mathrm{Z}, \\
\mathrm{mm}\end{array}$ \\
\hline $\mathrm{A} 01$ & -5.7 & -4.2 & 3.1 & 5.3 & 5.5 & 6.2 \\
\hline $\mathrm{A} 02$ & 4.4 & -1.1 & -4.2 & 5.3 & 5.4 & 5.6 \\
\hline $\mathrm{A} 03$ & 3.9 & -1.5 & 1.0 & 5.3 & 5.4 & 5.4 \\
\hline A04 & 1.4 & -2.0 & -1.6 & 5.4 & 5.4 & 5.6 \\
\hline A05 & 2.8 & 3.5 & 1.3 & 5.6 & 5.4 & 6.1 \\
\hline A06 & 2.7 & 1.1 & -1.1 & 5.4 & 5.2 & 5.5 \\
\hline A07 & -2.5 & 0.6 & 0.6 & 5.4 & 5.2 & 5.8 \\
\hline A08 & 2.2 & -1.9 & -1.3 & 5.4 & 5.2 & 5.8 \\
\hline A09 & -1.5 & 2.1 & -1.5 & 5.4 & 5.2 & 5.4 \\
\hline A10 & -4.2 & -0.4 & 0.9 & 5.4 & 5.4 & 5.5 \\
\hline A11 & -3.8 & 0.6 & 1.5 & 5.4 & 5.5 & 5.6 \\
\hline A12 & -2.6 & -5.6 & 1.8 & 5.3 & 5.5 & 5.5 \\
\hline A13 & -2.6 & -0.3 & 0.7 & 5.3 & 5.4 & 5.7 \\
\hline A14 & 10.9 & 5.4 & 0.8 & 5.4 & 5.4 & 5.9 \\
\hline A15 & 1.1 & 1.7 & -2.5 & 5.6 & 5.5 & 5.9 \\
\hline A16 & 4.2 & 0.2 & -7.3 & 5.7 & 5.4 & 5.7 \\
\hline A17 & -4.6 & -5.8 & 3.8 & 5.5 & 5.2 & 5.5 \\
\hline A18 & -2.3 & -0.2 & 3.8 & 5.4 & 5.2 & 5.4 \\
\hline A19 & -0.7 & -0.3 & 3.1 & 5.4 & 5.2 & 5.8 \\
\hline A20 & -1.9 & 4.1 & -1.9 & 5.5 & 5.5 & 5.8 \\
\hline A21 & -1.2 & 4.0 & -1.1 & 5.5 & 5.5 & 5.7 \\
\hline
\end{tabular}

Table 2. Deviations of control targets coordinates after transformation 
After transformation parameters determining the parameters were applied to Matterport point cloud. In order to check the quality of transformation the deviations $\varepsilon \mathrm{X}, \varepsilon \mathrm{Y}, \varepsilon_{\mathrm{Z}}$ and the accuracy of transformation for control targets have been determined (Table 2).

Transformation accuracy for control targets satisfies to TLS accuracy. To have a correct understanding about presented above results, the deviations of coordinates have been presented in the form of a vector field in Fig. 7.

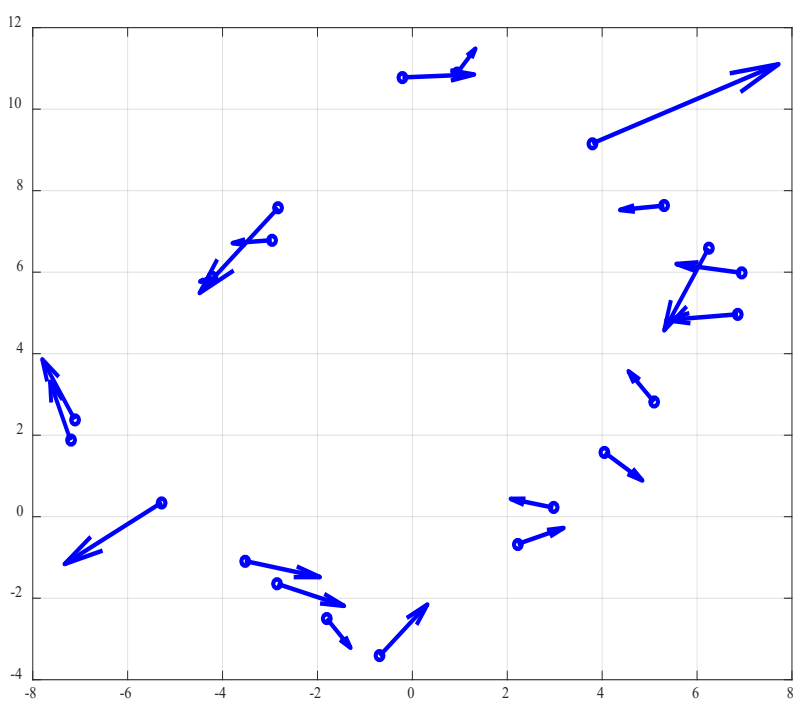

Figure 7. Vector plot of control targets coordinates deviations after transformation

From Fig. 7 we can conclude that deviations at control points obey to random law, there is no obvious systematic shift in coordinates. Once the scale correction is determined it is possible to apply Matterport data for real projects of industrial archaeology.

\section{QUINCY MINE}

As an object of industrial archaeology research, the Quincy Mine Hoist Engine was chosen. The Quincy Mining Company (QMC) was formed in 1846, with the main shaft at Upper Peninsula Michigan in the U.S. (Fig. 8).

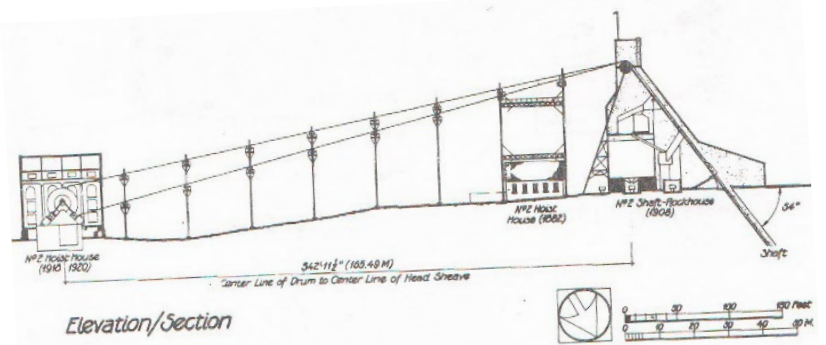

Figure 8. General view of the Quincy Mine, 1882-1920 (Quincy Mining Company HAER, 2019)

From 1859 until 1862 QMC evolved from small shaft to productive mine. The company built the first complete physical plant and in 1862 produced 2.1 million pounds of copper. From 1868 until 1920 QMC labour force grew from 500 to 2000 and produced 22 million pounds of copper per year. QMC technology passed from a pre-mechanized into a highly mechanized era. QMC had six shafts in operation at once, which were served by one of the largest in the world massive steam-powered hoist Nordberg Steam Hoist (Quincy Mining Company HAER, 2019).

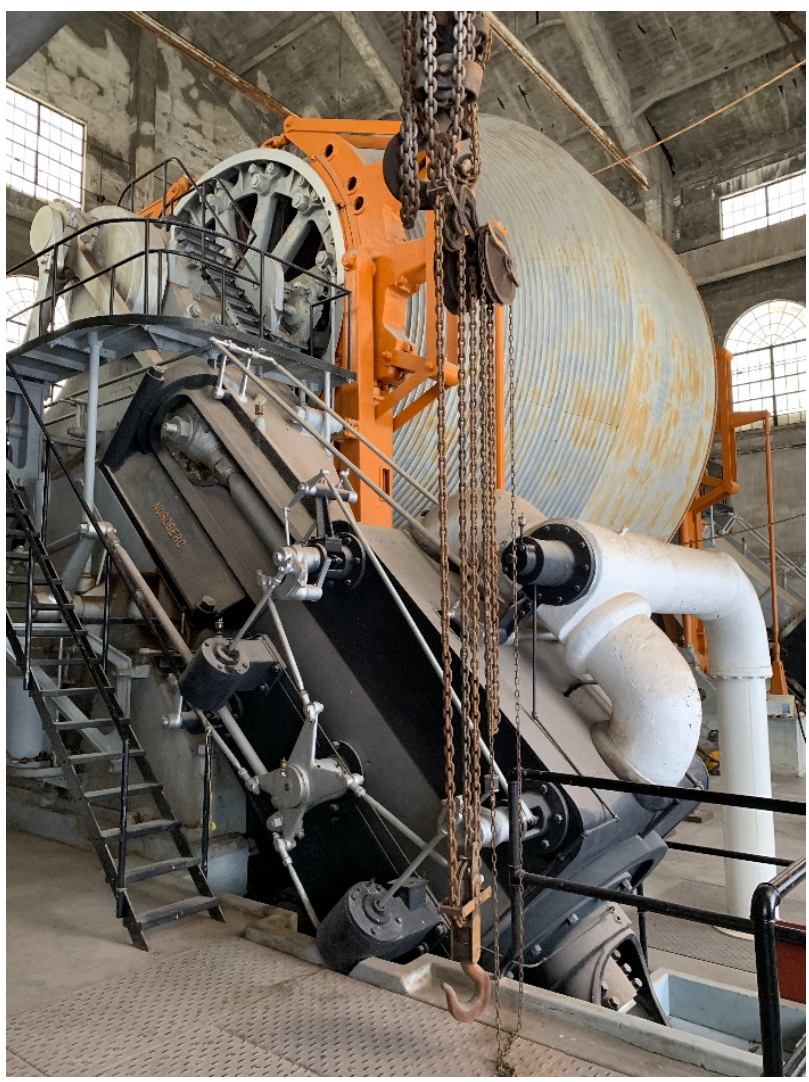

Figure 9. Current view of the Quincy Mine Hoist Engine

The last mining was performed in 1945 and shaft was closed. Since that time, the Quincy Mine as a complex of mining buildings and engines became an object of industrial archaeology. In 1978, the Historic American Engineering Record (HAER) documented the Quincy Mine as a significant engineering and industrial object in the U.S. An edition of the architecture and communities of the Quincy Mining Company was published.

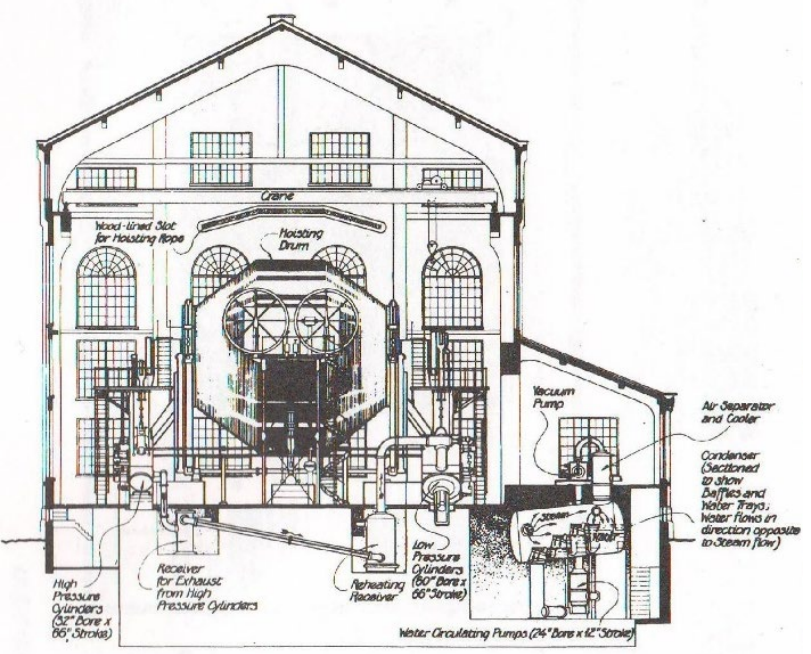

Figure 10. Cross section of the Quincy Mine Hoist Engine house, 1921 (Quincy Mining Company HAER, 2019)

Later, a museum at the shaft territory and many tourist tours have been organized. As it was mentioned, a goal of the research was a support of the QMHA with 3D model of Nordberg Steam Hoist 
and virtual reality (VR) tour. For that purpose, the Matterport was used.

\section{DATA ACQUISITION AND MODELING}

Data acquisition process can be divided into two steps. At the first step, data for the Hoist Engine house 3D model were acquired. A conventional photogrammetric procedure for outdoor modelling has been used, with photogrammetric data modelling with using of Agisoft Metashape software. The results of the modelling were badly needed further for virtual tour creation. Photogrammetric 3D model placed in the topographical landscape is presented in Fig. 11.

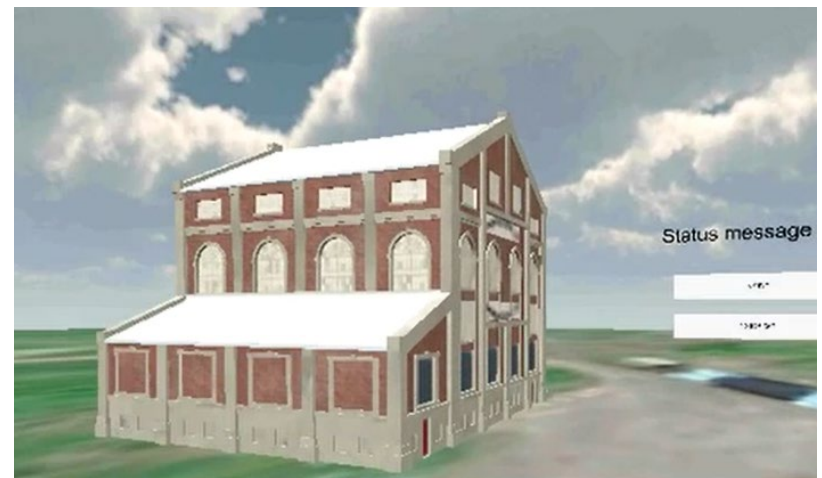

Figure 11. Hoist Engine house 3D model

At the second step, the indoor data acquisition was done by Matterport (Fig. 12). It is worth to mention that Nordberg Steam Hoist has a height over $12 \mathrm{~m}$ and it is a big challenge for Matterport. Another one problem it is inclement weather conditions during scanning. During data capturing the temperature was below $15 \mathrm{C}^{\circ}$. Inspite of that the Matterport managed with these critical conditions pretty good.

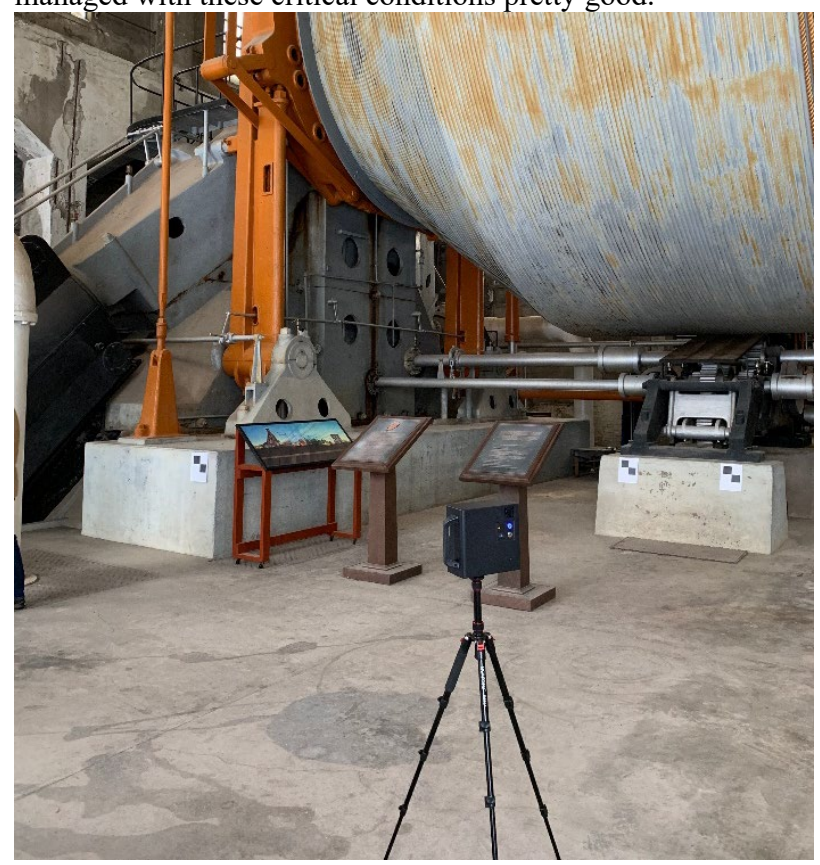

Figure 12. Data acquisition by Matterport

The scanning was accomplished on the ground from eight stations; the total size of the point cloud is almost 7 million points (Fig. 13).

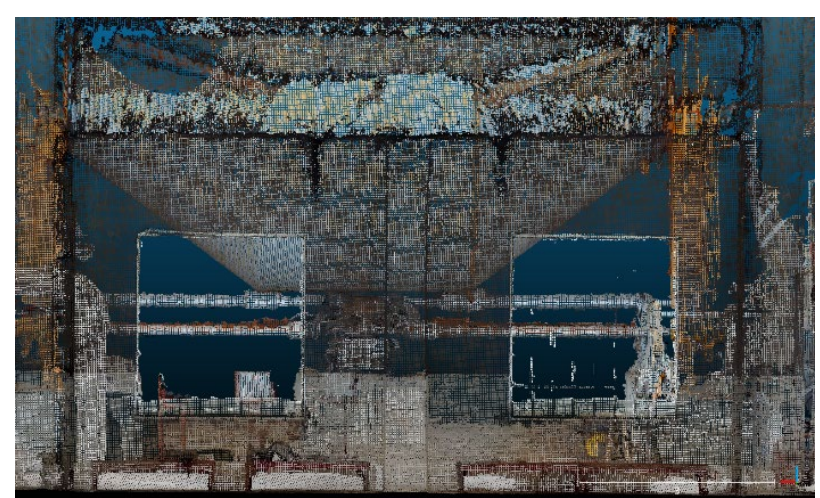

Figure 13. Matterport point cloud

In order to be sure that our data are correct, i.e. without the scale distortion, control targets were placed on the walls of the Hoist Engine house. Using of CloudCompare software eight distances between these targets were measured on Matterport point cloud (Fig. 14).

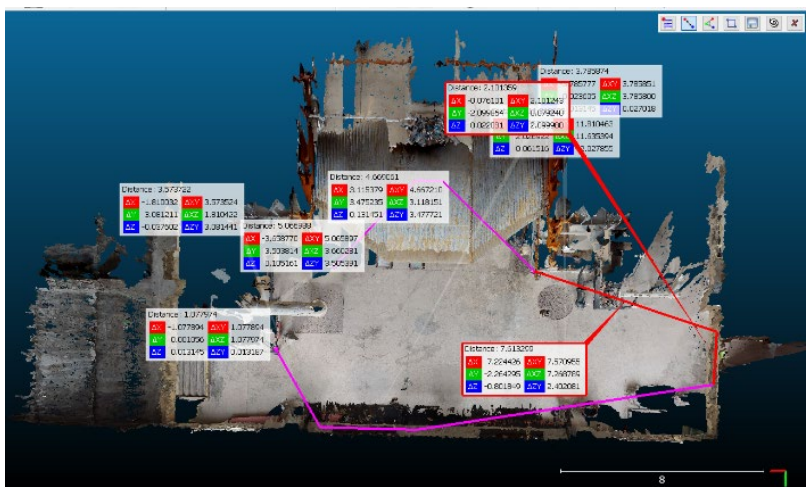

Figure 14. Measured control distances on Matterport point cloud

The same distances were measured by tape. The results of measurements and their comparison to tape measurements are presented in Table 3.

\begin{tabular}{|c|c|c|c|}
\hline Distances & Tape, $\mathrm{m}$ & Matterport, $\mathrm{m}$ & Differences, $\mathrm{m}$ \\
\hline $1-2$ & 1.080 & 1.092 & -0.012 \\
\hline $2-3$ & 4.670 & 4.700 & -0.029 \\
\hline $5-4$ & 4.096 & 4.039 & 0.057 \\
\hline $6-5$ & 3.573 & 3.581 & -0.008 \\
\hline $6-7$ & 3.786 & 3.810 & -0.024 \\
\hline $8-7$ & 11.811 & 11.887 & -0.076 \\
\hline $9-8$ & 2.101 & 2.134 & -0.033 \\
\hline $1-11$ & 5.067 & 5.105 & -0.038 \\
\hline
\end{tabular}

Table 3. Control distances and differences

Calculating the scale factor from those measurements is easy. Scale factor equals to 0.9960 . This value almost coincides with the value from paragraph 3 . After correction for the scale factor, the geometrically correct 3D model was got.

The final step of our research is a combination of the outside photogrammetric model with inside Matterport model and placement this model on the georeferenced map (Fig. 15). 


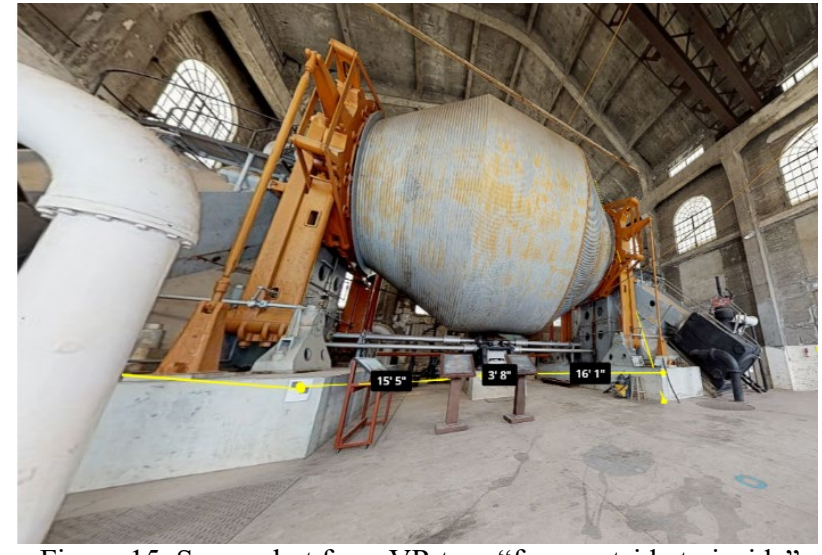

Figure 15. Screenshot from VR tour "from outside to inside"

The demo of our VR application was released using Unity3D software. As a result, any user can walk around and thru the Hoist Engine house and even make some measurements.

\section{CONCLUSIONS}

We have started our research from the reference to work (Palmer, 1989). Now it is clear how many technologies and its opportunities have changed since that time. The presented paper has proved that fact that new technologies superseded the old ones. Among such new technologies, a new 3D camera Matterport. Recording the industrial archaeology objects always was a challenge, first of all, due to a huge number of small and complex elements and details. These features are peculiar to the most industrial archaeology objects as mills, shafts, machinery, etc. It is found out that Matterport device allows collecting and modelling such data with good quality and high speed. Among other advantages it worth to mention ease-of-use and simple data integration into state-of-the-art VR software. However, in order to achieve better results much have to be done. By our research, the systematic errors in Matterport sensor allow achieving required accuracy (several centimetres level) for the BIM applications without preliminary calibration and correction. But, you have to be positive about your data. So that, anyway a check procedure is always needed. To achieve construction engineering accuracy (sub-centimetre level), which sometimes needs industrial archaeology for the documentary, the calibration procedure has to be developed and applied. It is badly needed to study the camera geometry more deeply and probably develop improved calibration model.

Summarizing we can conclude that nowadays geospatial technologies and software toolsets allow to build up 3D/4D geospatial datasets integrating topographical data, outdoor and indoor models. Archaeologists' and architects' dream to "fly from space and see a door knob" is a state-of-the-art.

\section{ACKNOWLEDGEMENTS}

Authors would like to say thank you to the Quincy Mine Hoist Association and museum authority for the given opportunity to carry out this research.

\section{REFERENCES}

Agostinelli, M., Clini, P., Lancioni, N., Quattrini, R., Sabbatini, G. 2007. The Volponi's kiln in Urbino. Industrial archeology and historic landscape in the cradle of the Renaissance. Documentation, survey and drawing. In Proc. XXI International CIPA Symposium, 178-183.
Barazzetti, L., Previtali, M., Roncoroni, F., 2018. Can we use Low-Cost 360 Degree Cameras to create accurate 3D models? In Proc. ISPRS Arch. Photogramm. Remote Sens. Spatial Inf. Sci., Vol. XLII-2, pp. 69- 75. https://doi.org/10.5194/isprs-archivesXLII-2-69-2018

Costantino, D., Angelini, M.G., Caprino, G. 2010. Laser scanner survey of an archaeological site - Scala Di Furno (Lecce, Italy). In Proc. ISPRS Arch. Photogramm. Remote Sens. Spatial Inf. Sci., Vol. XXXVIII, Part 5, 178-183.

Gärdin, D., Jimenez, A., 2018. Optical methods for 3Dreconstruction of railway bridges Infrared scanning, Close range photogrammetry and Terrestrial laser scanning, Department of Civil, Environmental and Natural Resources Engineering Luleå University of Technology, http://tu.divaportal.org/smash/get/diva2:1184613/FULLTEXT01.pdf

Gruenkemeier, A., 2008. 3D-documentation technologies for use in industrial archaeology applications. In Proc. ISPRS Arch. Photogramm. Remote Sens. Spatial Inf. Sci., Vol. XXXVII. Part B5, 291-296.

JAG3D, 2019. Java·Applied·Geodesy·3D, Steinbeis Transfer Centre Applied Geodesy, https://software.applied-geodesy.org [Accessed: 11th March 2019]

Khoshelham, K., Elberink, S.O., 2012. Accuracy and resolution of Kinect depth data for indoor mapping applications. Sensors, 12(2), 1437-1454. doi:10.3390/s120201437

Lehtola, V. V., Kaartinen H., Nuchter A., Kaijaluoto R., Kukko A., Litkey P., Honkavaara E., Rosnell T., Vaaja M. T., Virtanen J. P., Kurkela M., El Issaoui A., Zhu L., Jaakkola A., Hyyppa J., 2017. Comparison of the selected state-of-the-art 3D indoor scanning and point cloud generation methods. Remote Sensing, 9(8), 796. https://doi.org/10.3390/rs9080796

Matterport, 2019a. Matterport Pro 3D Camera specifications. https://matterport.com/pro2-3d-camera/ [Accessed: 11th March 2019].

Matterport, 2019b. Scanning a Job Site for Construction Documentation. https://support.matterport.com/hc/enus/articles/115013811827-Scanning-a-Job-Site-for-

Construction-Documentation [Accessed: 11th March 2019].

Palmer, M., Neaverson, P. 1998. Industrial Archaeology. Principles and practice. London: Routledge.

Pulcrano, M., Scandurra, S., Minin, G., di Luggo, A., 2019. 3D cameras acquisitions for the documentation of cultural heritage. In Proc. ISPRS Arch. Photogramm. Remote Sens. Spatial Inf. Sci., XLII-2/W9, 639-646. https://doi.org/10.5194/isprs-archivesXLII-2-W9-639-2019

Quincy Mining Company HAER. 2019. A Look at the architecture and communities of the Quincy Mining Company, published by Quincy Mine Hoist Association (QMHA). https://quincymine.com [Accessed: 11th March 2019].

Virtanen, J.-P., Kurkela, M., Turppa, T., Vaaja, M.T., Julin, A., Kukko, A., Hyyppa, J., Ahlavuo, M., von Numers, J.E., Haggren, H., Hyyppa, H. 2018. Depth camera indoor mapping for 3D virtual radio play. The Photogrammetric Record 33(162), 171195. https://doi.org/10.1111/phor.12239 